\title{
Erratum to: Meiosis-I in Mesostoma ehrenbergii spermatocytes includes distance segregation and inter-polar movements of univalents, and vigorous oscillations of bivalents
}

Jessica Ferraro-Gideon • Carina Hoang • Arthur Forer

Published online: 1 October 2013

(C) Springer-Verlag Wien 2013

Erratum to: Protoplasma

DOI: $10.1007 / \mathrm{s} 00709-013-0532-9$

Unfortunately, the original publication of this article contains a mistake. Column 4 of Table 2, row 3 ("Combined") should read: $5.66 \pm 2.16$ [replacing $5.08 \pm 1.75$ ].

The online version of the original article can be found at http://dx.doi.org/ 10.1007/s00709-013-0532-9.

J. Ferraro-Gideon $\cdot$ C. Hoang $\cdot$ A. Forer $(\bowtie)$

Department of Biology, York University, 4700 Keele St, Toronto,

ON M3J 1P3, Canada

e-mail: aforer@yorku.ca

J. Ferraro-Gideon

e-mail: ferraroj@yorku.ca 